\title{
Outcomes of Primary Bidirectional Glenn in Children with Single Ventricle Physiology and Increased Pulmonary Blood Flow
}

\author{
Ahmed M. Dohain, MD, ${ }^{1,2}$ Muhammed A. Mashat, MBBS, ${ }^{3}$ Ahmed M.A Al-Mojaddidi, MRCPCH, ${ }^{1}$ \\ Mohamed E. Abdelmotaleb, MRCPCH, ${ }^{1}$ Abdulla A. Mashat, MBBS, ${ }^{3}$ Gaser Abdelmohsen, MD, ${ }^{1,2}$ \\ Mohamed H. Abdelsalam, MD, ${ }^{1,4}$ Jameel Al-Ata, MD, ${ }^{1}$ Wael A. Attia, MD, ${ }^{1,2}$ Mazen S. Faden, MD, ${ }^{5}$ \\ Zaher F. Zaher, MD, ${ }^{1}$ Saud A. Bahaidarah, MD,${ }^{1}$ Naif A. Alkhushi, FRCPC, ${ }^{1}$ Osman O. Al-Radi, FRCSC, ${ }^{3}$ \\ Ahmed A. Elassal, MD, 3,6 \\ ${ }^{1}$ Pediatric Cardiology Division, Department of Pediatrics, King Abdulaziz University, Jeddah, Saudi Arabia; ${ }^{2}$ Pediatric Cardiology \\ Division, Department of pediatrics, Cairo University, Cairo, Egypt; ${ }^{3}$ Cardiac Surgery Division, Department of Surgery, King \\ Abdulaziz University, Jeddah, Saudi Arabia; ${ }^{4}$ Cardiology Department, Benha University, Benha, Egypt; ${ }^{5}$ Department of Anesthesia, \\ King Abdulaziz University, Jeddah, Saudi Arabia; ${ }^{6}$ Department of Cardiothoracic Surgery, Zagazig University, Zagazig, Egypt
}

\section{ABSTRACT}

Background: We reported our experience in managing patients with single ventricle (SV) physiology and increased pulmonary blood flow (PBF), aiming to assess if it is feasible to proceed with primary Bidirectional Glenn (BDG) without a prior operation to limit PBF.

Materials and methods: This is a retrospective study with 51 consecutive patients who underwent BDG operation as a primary operation or a second stage prior to the definitive Fontan operation at King Abdulaziz University Hospital (KAUH) in Jeddah, Saudi Arabia between 2010 and 2018. Patients were categorized into two groups based on their PBF prior to the operation: Patients who had SV physiology and increased PBF (seven patients) vs. patients with SV physiology and restricted PBF (44 patients).

Results: The median age for the increased PBF group was 9.9 months [interquartile range (IQR): 2-16.9 months], and the median age for the restricted $\mathrm{PBF}$ group was 15.3 months (IQR: 6.7-42.6 months). Although the length of hospital stay was longer in patients with increased PBF $(P=$ 0.039 ), we couldn't find a statistically significant difference in early mortality, duration of mechanical ventilation, length of pleural drainage, and length of intensive care unit (ICU) stay between the groups.

Conclusion: In our experience, we found that primary BDG could be done safely for patients having SV physiology and increased $\mathrm{PBF}$ with acceptable short-term outcomes. It might further reduce the morbidity and mortality for those patients by avoiding the risk of initial pulmonary artery banding or aortopulmonary shunts.

Received August 29, 2020; accepted September 15, 2020.

Correspondence: Dr. Ahmed Mohamed Dohain, MD, Department of Pediatrics, King Abdulaziz University, Feddah, Saudi Arabia, P.O. Box 80215, 7eddab 21589, Saudi Arabia, Telephone +966541611496 (e-mail: adobain@yahoo.com; amdobain@kau.edu.sa)

\section{INTRODUCTION}

Bidirectional Glenn (BDG) operation has become an accepted intermediate-stage palliation for patients with single ventricle (SV) physiology, before the final Fontan procedure [Albanese 1992; Bridges 1990; Hawkins 1993; Hopkins 1985; Lamberti 1990; Pridjian 1993].

The principle of first-stage palliation, prior to BDG, of patients with SV cardiac anomalies is to achieve a systemic cardiac output free from obstruction, in addition to an unobstructed and controlled pulmonary blood flow (PBF), and an unobstructed pulmonary venous return [Alsoufi 2013; Rodefeld 2005].

BDG can be performed as a primary procedure in patients with naturally limited PBF. Patients who had a prior systemic pulmonary artery shunt operation or a pulmonary artery banding (PAB) operation, the BDG became the second operation [Warrier 2004].

In patients with SV anatomy and increased PBF, initial palliation typically has been PAB [Alsoufi 2013; Franklin 1991; Freedom 1986; Lee 2014; Rodefeld 2005; Warrier 2004]. Nonetheless, several centers reported disappointing short- and long-term outcomes of patients with SV anomalies typically palliated with $\mathrm{PAB}$, and some authors recommended the usage of an aortopulmonary shunt plus division of the main pulmonary artery (PA) as an alternative firststage palliation strategy, that would provide a source of $\mathrm{PBF}$ that is more reliable along with better pulmonary vascular protection [Bradley 2002; Franklin 1991; Freedom 1986].

On the other hand, first-stage palliation for SV physiology in which a systemic-to-pulmonary shunt is the main source of PBF still carries some of the highest morbidity and mortality rates related to congenital heart surgery [Tamisier 1990], due to shunt thrombosis or overcirculation.

In this study, we report our experience in managing patients with SV physiology and increased PBF, aiming to assess whether it is feasible to proceed with primary BDG without a prior operation to limit PBF. Additionally, we aim 
to evaluate the clinical characteristics, the outcomes and the factors affecting the outcomes for all patients who underwent BDG, during the study period at our institution.

\section{MATERIALS AND METHODS}

We retrospectively reviewed all patients who underwent BDG operation as a primary operation or a second stage prior to the definitive Fontan operation at King Abdulaziz University Hospital (KAUH) in Jeddah, Saudi Arabia between 2010 and 2018. Approval of this study was obtained from the Research Ethics Board at our institution, and consent for the surgical procedures was obtained from each patient. The requirement for individual consent was waived for this observational study.

Demographic, clinical, operative, catheterization, echocardiographic and outcome data were collected from hospital medical records and the cardiac surgical database.

The patients were categorized into two groups, based on their PBF prior to the operation: patients who had SV physiology and increased PBF versus patients with SV physiology and restricted PBF. The patients were considered to have increased PBF if they had clinical manifestation of heart failure, chest $\mathrm{x}$-ray was showing pulmonary congestion, and echocardiogram was revealing unobstructed PBF with pressure gradient less than $30 \mathrm{mmHg}$ across right ventricular outflow tract.

The findings from the most recent echocardiogram done before the BDG operation were reviewed. Atrioventricular valve (AVV) regurgitation was graded as severe, moderate, mild, or absent, based on pulse wave tracing and color Doppler mapping.

Data from the most recent cardiac catheterization performed before the BDG operation was reviewed. Measurements recorded included ventricular end-diastolic pressure (VEDP), mean PA pressure, indexed pulmonary vascular resistance (PVRI), and pulmonary venous wedge pressure. Preoperative and postoperative oxygen saturations were noted.

Surgical details were recorded, including cardiopulmonary bypass time (CPB) and aortic cross-clamp time. Also, we documented the procedures that were done prior to BDG procedure, such as the Norwood procedure, patent ductus arteriosus (PDA) stent, $\mathrm{PAB}$, or a previous BlalockTaussig (BT) shunt and simultaneous procedures done at the time of the BDG procedure, such as PA plasty, AVV repair and atrial septectomy.

Early postoperative hemodynamic data was noted, including superior vena cava (SVC) pressure (through internal jugular or subclavian central lines). Inotropes and vasoactive medications were recorded at an hourly interval during the first 24 hours after admission to postoperative intensive care unit (ICU). In the current analysis, the inotropic score (IS) was calculated as per Wernovsky [Wernovsky 1995] and the Vasoactive-Inotropic Score (VIS) as described by Gaies et al. [Gaies 2014] and Davidson et al. [Davidson 2012].
IS = Dopamine dose $(\mu \mathrm{g} / \mathrm{kg} / \mathrm{min})+$ Dobutamine dose $(\mu \mathrm{g} / \mathrm{kg} / \mathrm{min})+100 \times$ epinephrine dose $(\mu \mathrm{g} / \mathrm{kg} / \mathrm{min})$

VIS $=$ IS + $10 X$ Milrinone dose $(\mu \mathrm{g} / \mathrm{kg} / \mathrm{min})+10,000 \times$ Vasopressin dose $(\mathrm{U} / \mathrm{kg} / \mathrm{min})+100 \times$ Norepinephrine dose $(\mu \mathrm{g} / \mathrm{kg} / \mathrm{min})$

Postoperative outcome data included the total duration of mechanical ventilation, duration of pleural drainage, ICU stay, total hospital stay, VIS, morbidity, and mortality. The duration of chest tube drainage was determined by the day on which all the intraoperative chest tubes were removed. Morbidity was determined by the existence of a complication, such as early postoperative intervention, delayed chest closure, diaphragmatic paralysis and chylous effusion that required dietary changes to a low-fat diet.

Statistical analysis: Statistical analysis using "IBM SPSS statistics ver. 20.0" and "Excel 2013" was applied to test the hypothesis. Descriptive statistics were done to calculate means, Std. Deviation, median and interquartile range (IQR). Shapiro-Wilk test was used to test the normality of the study sample variables. Pearson's correlation was used to identify the strength and direction of relationship that present between two or more variables. Independent Sample T-Test / Mann-Whitney tests were applied to find if there was a significant difference between two groups and Chi-square test was used to find the significant association between categorical variables.

\section{RESULTS}

Patients characteristics: Fifty-one consecutive patients underwent BDG at KAUH from 2010 to 2018. The median age at the time of BDG was 15.2 months (IQR: 6-41.8 months), and the median weight was $8 \mathrm{~kg}$ (IQR: $5.9-11 \mathrm{~kg}$ ). Six patients $(11.8 \%)$ were less than four months of age and three patients $(5.9 \%)$ were less than $4 \mathrm{~kg}$ at the time of the operation.

Preoperative evaluation: The most common anatomic subtypes were tricuspid atresia in $23.5 \%$, unbalanced atrioventricular septal defect (UAVSD) in $17.6 \%$, double outlet right ventricle (DORV) in $17.6 \%$, double inlet left ventricle (DILV) in $11.8 \%$, hypoplastic left heart syndrome (HLHS) in $5.9 \%$, or other in $23.5 \%$. Patient diagnoses are summarized in Table 1. (Table 1)

Twenty-seven patients $(52.9 \%)$ had no previous palliation, whereas the remaining 24 patients $(47.1 \%)$ had a previous palliation, such as PDA stent $(N=12)$, Norwood operation $(N=6)$, aortopulmonary shunt $(N=3), \mathrm{PAB}(N=2)$, and atrial septostomy $(N=1)$.

Anatomically, 20 patients (39.2\%) had a morphologic single left ventricle (LV), 14 patients $(27.5 \%)$ had a morphologic single right ventricle (RV), and 17 patients $(33.3 \%)$ had either two ventricles that could not be partitioned or indeterminate SV morphology. AVV function was normal in $72.5 \%$, whereas insufficiency was identified as mild in $13.7 \%$, moderate in $9.8 \%$, and severe in $3.9 \%$.

Preoperative catheterization was performed in 34 patients (66.7\%). Median PA pressure was $18 \mathrm{mmHg}$, median PVRI 
Table 1. Preoperative data of patients who underwent bidirectional Glenn

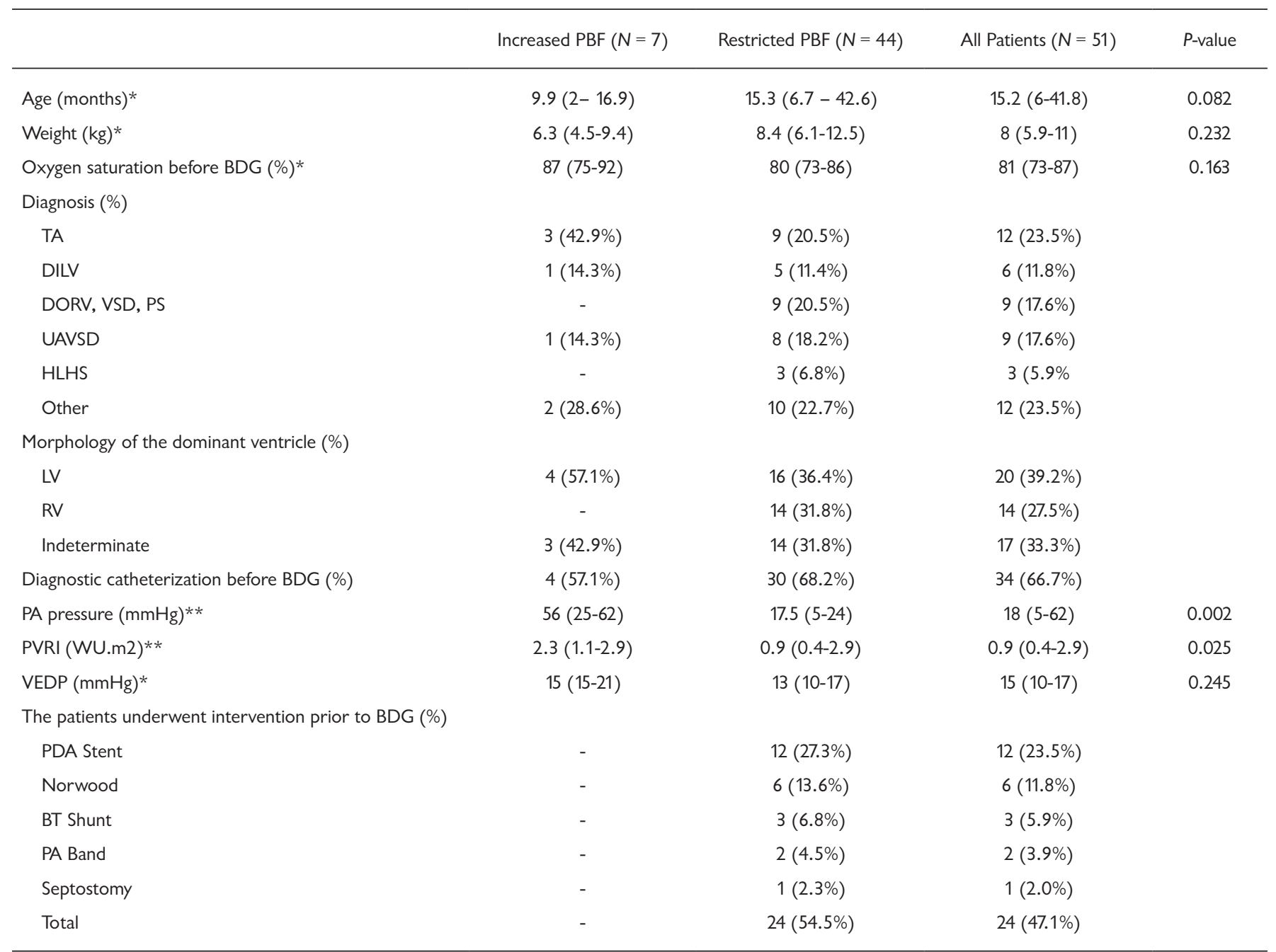

BDG, bidirectional Glenn; BT, Blalock-Taussig; DILV, double inlet left ventricle; DORV, double outlet right ventricle; HLHS, hypoplastic left heart syndrome; PA, pulmonary artery; PBF, pulmonary blood flow; PDA, patent ductus arteriosus; PS, pulmonary stenosis; PVRI, pulmonary vascular resistance index, RV right ventricle; TA, tricuspid atresia; UAVSD, unbalanced atrioventricular septal defect; VEDP, ventricular end-diastolic pressure, WU; Woods unit. *Median (interquartile range); **Median (minimum-maximum)

was 0.9 Woods unit (WU).m2 and median VEDP was 15 mmHg (IQR: 10-17 mmHg).

Operative data: Forty patients underwent unilateral BDG, whereas bilateral BDG was performed in 10 patients with bilateral SVC and one patient had Kawashima procedure. The most common additional procedures performed included atrial septectomy $(N=20)$, pulmonary arterioplasty $(N=14)$, AVV repair $(N=2)$, ventricular septal defect (VSD) enlargement $(N=1)$ and total anomalous pulmonary venous return (TAPVR) repair $(N=3)$. The operative details for each group are represented in Table 2. (Table 2)

Outcome data: Hemodynamic studies showed a median postoperative SVC pressure of $18 \mathrm{mmHg}$ (IQR: $13-21 \mathrm{mmHg}$ ). Arterial oxygen saturation rose from 81\% (IQR: 73-87\%) preoperatively to $86 \%$ (IQR: $84-90 \%$ ) postoperatively.
The median postoperative VIS was 9.5 on postoperative 24 hours. The median duration of pleural drainage was five days (IQR: 4-7 days). The median duration of mechanical ventilation was 10 hours (IQR: 6 -23.75 hours). The median duration of stay in the ICU and hospital was four days (IQR: 2-6.75 days) and eight days (IQR: 6-13 days), respectively.

Three patients needed early postoperative interventional cardiac catheterization, one patient for Glenn stenting, one patient for right pulmonary artery stenting, and another patient for small left SVC coil occlusion. In addition, three patients needed surgical intervention for PAB tightening, plication of the diaphragm, and permanent pacemaker insertion. Two patients had delayed chest closure. Postoperatively, eight patients had chylous effusion (15.7\%), and three patients had diaphragmatic paralysis (5.9\%). One of the patients needed 
Table 2. Operative details of patients who underwent bidirectional Glenn ${ }^{a}$

\begin{tabular}{lccc}
\hline & Increased PBF $(N=7)$ & Restricted PBF $(N=44)$ & All Patients $(N=51)$ \\
\hline Type of BDG & & & \\
Right BDG & $7(100 \%)$ & $33(75 \%)$ & $40(78.4 \%)$ \\
Bilateral BDG & - & $10(22.7 \%)$ & $10(19.6 \%)$ \\
Kawashima & - & $1(2.3 \%)$ & $1(2 \%)$ \\
Additional procedures & - & & $14(27.5 \%)$ \\
PA Plasty & $4(57.1 \%)$ & $14(31.8 \%)$ & $20(39.2 \%)$ \\
Septectomy & - & $16(36.4 \%)$ & $2(3.9 \%)$ \\
AVV repair & - & $2(4.5 \%)$ & $1(2.0 \%)$ \\
VSD enlargement & - & $1(2.3 \%)$ & $3(5.9 \%)$ \\
TAPVR repair & $40(21-41)$ & $3(6.8 \%)$ & $52(42-68)$ \\
Bypass Time (min) & $21(0-38)$ & $53(44-72)$ & $16.5(0-38)$ \\
Cross-clamp time (min) & $6(0-40)$ & 0.027 \\
\hline
\end{tabular}

AVV, atrioventricular valve; BDG, bidirectional Glenn; PA, pulmonary artery; PBF, pulmonary blood flow; TAPVR, total anomalous pulmonary venous return; VSD, ventricular septal defect. ${ }^{a}$ Continuous variables are presented as median (interquartile range).

diaphragmatic placation. Early postoperative mortality following BDG was two patients (3.9\%).

The PA pressure had significant positive correlation with the duration of pleural drainage $(P=0.013)$, but it was not correlated with the duration of mechanical ventilation, the length of ICU stay, or hospital stay. VIS at 24 hours postoperatively was positively correlated with duration of mechanical ventilation $(P=0.013)$ and ICU length of stay $(P=0.029)$.

Two group comparison: Among the 51 patients who underwent BDG procedure, seven patients had increased $\mathrm{PBF}$ at the time of the surgery and a primary BDG was performed for them. The remaining 44 patients had restricted $\mathrm{PBF}$ as an initial congenital cardiac lesion or after previous surgical intervention like PAB, BT shunt or Norwood procedure. (Table 1)

The median age for the increased PBF group was 9.9 months (IQR: 2-16.9 months), the age of three out of seven patients in this group was less than three months at the time of BDG (35, 62 and 78 days). On the other hand, the median age for the restricted PBF group was 15.3 months (IQR: 6.742.6 months).

In SV physiology and increased PBF group, four patients had cardiac catheterization before BDG procedure. The other three patients, who were less than three months old, had manifestations of heart failure, high oxygen saturations and lung plethora (in chest $\mathrm{x}$-ray) which indicate low PVR. Accordingly, they underwent primary PBG based on the clinical evaluation without cardiac catheterization.

The PA pressure was significantly higher in patients with increased PBF than those with restricted PBF ( $P$-value was 0.002). The median PVRI in the patients with restricted PBF was 0.9 WU.m ${ }^{2}$ (range from 0.4 to 2.9 WU.m²), and the median PVRI for patients with increased PBF was 2.3 WU.m ${ }^{2}$ (range from 1.1 to $2.9 \mathrm{WU} \cdot \mathrm{m}^{2}$ ), which is significantly higher than patients with restricted PBF ( $P$-value was 0.025$)$.
We compared the short-term outcome of the two groups, and we found that initial ICU hemodynamic data was very similar. Median postoperative SVC pressure was $17 \mathrm{mmHg}$ (IQR: $10-23 \mathrm{mmHg}$ ) in the increased PBF group and 18 mmHg (IQR: $13-21 \mathrm{mmHg}$ ) in the restricted PBF group $(P$ $=0.85)$. Median postoperative arterial oxygen saturation was 86\% (IQR: $84-94 \%$ ) and 87\% (IQR: 84-90\%) in increased $\mathrm{PBF}$ group and restricted $\mathrm{PBF}$ group, respectively $(P=0.555)$.

The maximum VIS after 24 hours postoperatively was not significantly different in both groups, $P$-value was 0.473. Postoperatively, one patient from each group needed inhaled nitric oxide as a pulmonary vasodilator. In addition, one patient from each group had delayed chest closure. Throughout the hospital stay, two patients (28.6\%) from the increased $\mathrm{PBF}$ group developed postoperative chylous drainage and six patients (13.6\%) from the restricted PBF group.

There was one postoperative mortality from each group; this does not represent a statistical significance. The data for duration of mechanical ventilation, length of ICU stay, length of hospitalization, and length of pleural drainage are presented in Table 3. (Table 3) Although the length of hospital stay was longer in the increased PBF group $(P=0.039)$, we could not find a statistically significant difference between the duration of mechanical ventilation, length of pleural drainage, and length of ICU stay in both groups.

\section{DISCUSSION}

Patients with various SV anomalies associated with increased PBF require early control with $\mathrm{PAB}$ to prevent pulmonary overcirculation and the later development of pulmonary vascular disease [Alsoufi 2015]. Freedom and coworkers 
Table 3. The outcome variables ${ }^{a}$

\begin{tabular}{lccc}
\hline Variable & Increased PBF & Restricted PBF & P-value \\
\hline Duration of pleural drainage (days) & $9.0(4.0-15.0)$ & $5.0(4.0-6.0)$ & 0.060 \\
Duration of mechanical ventilation (hours) & $15.5(7.5-92.3)$ & $8.5(6.0-24.0)$ & 0.531 \\
Length of ICU stay (days) & $5.5(3.5-11.0)$ & $4.0(2.0-6.3)$ & 0.207 \\
Length of hospital stay (days) & $17.0(9.0-22.8)$ & $8.0(6.0-11.0)$ & 0.039 \\
\hline
\end{tabular}

PBF, pulmonary blood flow. ${ }^{a}$ Continuous variables are presented as median (interquartile range).

[Freedom 1987] demonstrated a high incidence of systemic outflow obstruction in patients with SV treated initially with PAB placement [Ilbawi 1991; Franklin 1990; Lee 2014; Lui 1993]. The advantages of this strategy are mainly the avoidance of CPB in the neonatal period and it decreases risk of thrombotic occlusion than might occur with a BT shunt [Alsoufi 2015].

However, there are several disadvantages of $\mathrm{PAB}$ due to the difficulty obtaining adequate protection of pulmonary circulation with $\mathrm{PAB}$, the possibility of distortion of PA and damage of the pulmonary valve with subsequent regurgitation. It also increases ventricular hypertrophy that might accelerate bulboventricular foramen rate of narrowing with subsequent early development of systemic ventricle outflow tract obstruction [Alsoufi 2013; Franklin 1991; Fraser 2009; Freedom 1986; Lee 2014; Matitiau 1992]. Also, reported hospital mortality after PAB continues to be high; in a recent Society of Thoracic Surgeons harvest, hospital mortality for PAB was $8.9 \%$ [Alsoufi 2015].

Performing an early primary BDG for patients with SV and increased PBF could be an option to provide a low pressure PBF and avoid the risk of steal from the systemic circulation that might happen in patients with parallel circulation such as those with PAB or aortopulmonary shunts [Alsoufi 2012]. In addition, it will help in avoiding the risk of firststage palliation early in life as PAB or BT shunt.

In this series, we studied the short-term outcome of patients who had SV with increased PBF and underwent a primary BDG in comparison with those who had SV and restricted $\mathrm{PBF}$ as an initial congenital lesion or after previous palliative procedure, such as PAB, aortopulmonary shunt, Norwood procedure, or PDA stent. We found that early mortality, mechanical ventilation duration, pleural drainage duration and ICU length of stay were not significantly different in both groups but the length of hospital stay was longer in patients who had SV with increased PBF and underwent a primary BDG $(P=0.039)$.

Our practice is making a concentrated effort to wean patients off any oxygen supply before hospital discharge, which might result in prolonged hospital stay in the group of increased PBF who may require oxygen supplementation for longer duration due to preoperative congested lungs. The preoperative PA pressure was higher in the patients' group with increased PBF, which could be flow related. Postoperatively, SVC pressure and oxygen saturation were comparable between both groups.

Taking into consideration that the comparison was made between the outcomes of BDG only and not including the duration of pleural drainage, duration of mechanical ventilation, ICU stay, hospital stay and mortality of the procedures prior to BDG in the restricted PBF group. Therefore, the cost and risk of the first-stage palliation should be considered in comparing the outcomes of both groups, in terms of cost benefit.

We performed further analysis of variables that might affect the outcomes, following the BDG operation for the overall patients' population. We found that preoperative PA pressure has significant association with the duration of pleural drainage $(P=0.013)$, but not affecting the other outcomes.

The maximum VIS calculated in the first 24 hours after ICU admission was studied by Gaies et al., and they found it significantly and strongly associated with morbidity and mortality in this multi-institutional cohort of infants after cardiac surgery [Gaies 2014]. In the present study, the maximum VIS at 24 hours postoperatively had significant correlation with duration of mechanical ventilation and ICU stay $(P=0.013$ and $P=0.029$, respectively). On the other hand, the VEDP, ventricular morphology, degree of AAV regurgitation and CPB time were not associated with BDG outcomes.

The variables associated with surgical outcomes have been addressed mainly by single-center studies with varying and sometimes conflicting results. Those studies have identified age, weight-for-age, PA pressure, ventricular morphology, AVV regurgitation, and $\mathrm{CPB}$ as factors associated with BDG outcomes [Anderson 2009; Dohain 2020; Elassal 2020; Jaquiss 2006; Kogon 2008; Petrucci 2010].

Study limitations: The retrospective nature of the study was a limitation to get all the required data. The group of patients who had SV physiology with increased PBF was relatively small, which limits the statistical power of the study. Because of difficulties attributable to vascular access or clinically based decisions, some patients were not catheterized, and catheterization data variables are not available for all patients.

\section{CONCLUSION}

There is limited published data showing the outcomes of primary BDG for patients with SV physiology and increased 
PBF. In our experience, we found that it can be done safely with acceptable short-term outcomes. Even more, it may reduce the morbidity and mortality for those patients by skipping the first-stage palliation as initial PAB or aortopulmonary shunts. Although, we did not find much difference in the outcome after BDG between increased PBF group and restricted PBF group, but we cannot always advocate avoiding $\mathrm{PAB}$ and proceeding with primary $\mathrm{BDG}$ directly due to limited data. We recommend future studies to evaluate shortand long-term outcomes of such patients.

\section{REFERENCES}

Albanese SB, Carotti A, Di Donato RM, et al. 1992. Bidirectional cavopulmonary anastomosis in patients under two years of age. J Thorac Cardiovasc Surg 104:904-909.

Alsoufi B. 2013. Management of the single ventricle and potentially obstructive systemic ventricular outflow tract. J Saudi Hear Assoc 25:191-202.

Alsoufi B, Manlhiot C, Awan A, et al. 2012. Current outcomes of the Glenn bidirectional cavopulmonary connection for single ventricle palliation. Eur J Cardiothorac Surg 42:42-49.

Alsoufi B, Manlhiot C, Ehrlich A, et al. 2015. Results of palliation with an initial pulmonary artery band in patients with single ventricle associated with unrestricted pulmonary blood flow. J Thorac Cardiovasc Surg $149: 213-220$

Anderson JB, Beekman RH 3rd, Border WL, et al. 2009. Lower weightfor-age z score adversely affects hospital length of stay after the bidirectional Glenn procedure in 100 infants with a single ventricle. J Thorac Cardiovasc Surg 138:397-404.

Bradley SM, Simsic JM, Atz AM, Dorman BH. 2002. The infant with single ventricle and excessive pulmonary blood flow: results of a strategy of pulmonary artery division and shunt. Ann Thorac Surg 74:805-10; discussion 810

Bridges ND, Jonas RA, Mayer JE, et al. 1990. Bidirectional cavopulmonary anastomosis as interim palliation for high-risk Fontan candidates. Early results. Circulation 82:IV170-6.

Davidson J, Tong S, Hancock H, et al. 2012. Prospective validation of the vasoactive-inotropic score and correlation to short-term outcomes in neonates and infants after cardiothoracic surgery. Intensive Care Med 38:1184-1190.

Dohain AM, Ismail MF, Elmahrouk AF, et al. 2020. The outcomes of bidirectional Glenn before and after 4 months of age: A comparative study. J Card Surg. https://doi.org/10.1111/jocs.15055.

Elassal AA, Al-Radi OO, Dohain AM, et al. 2020. Excess nonhemorrhagic pleural drainage after surgery for congenital heart diseases: Single center experience. J Card Surg 35:108-112. https://doi.org/10.1111/ jocs. 14338 .

Franklin R, D. Sullivan I, H. Anderson R, et al. 1990. Is banding of the pulmonary trunk obsolete for infants with tricuspid atresia and double inlet ventricle with a discordant ventriculoarterial connection? Role of aortic arch obstruction and subaortic stenosis. J Am Coll Cardiol. 16:1455-1464.

Franklin RC, Spiegelhalter DJ, Anderson RH, et al. 1991. Double-inlet ventricle presenting in infancy. II. Results of palliative operations. J Thorac Cardiovasc Surg 101:917-923.
Fraser CDJ. 2009. Management of systemic outlet obstruction in patients undergoing single ventricle palliation. Semin Thorac Cardiovasc Surg Pediatr Card Surg Annu 70-75.

Freedom RM. 1987. The dinosaur and banding of the main pulmonary trunk in the heart with functionally one ventricle and transposition of the great arteries: a saga of evolution and caution. J Am Coll Cardiol $10: 427-429$.

Freedom RM, Benson LN, Smallhorn JF, et al. 1986. Subaortic stenosis, the univentricular heart, and banding of the pulmonary artery: an analysis of the courses of 43 patients with univentricular heart palliated by pulmonary artery banding. Circulation 73:758-764.

Gaies MG, Jeffries HE, Niebler RA, et al. 2014. Vasoactive-inotropic score is associated with outcome after infant cardiac surgery: an analysis from the Pediatric Cardiac Critical Care Consortium and Virtual PICU System Registries. Pediatr Crit Care Med 15:529-537.

Hawkins JA, Shaddy RE, Day RW, et al. 1993. Mid-term results after bidirectional cavopulmonary shunts. Ann Thorac Surg 56:833-837.

Hopkins RA, Armstrong BE, Serwer GA, et al. 1985. Physiological rationale for a bidirectional cavopulmonary shunt. A versatile complement to the Fontan principle. J Thorac Cardiovasc Surg 90:391-398.

Ilbawi MN, DeLeon SY, Wilson WRJ, et al. 1991. Advantages of early relief of subaortic stenosis in single ventricle equivalents. Ann Thorac Surg 52:842-849.

Jaquiss RDB, Siehr SL, Ghanayem NS, et al. 2006. Early cavopulmonary anastomosis after Norwood procedure results in excellent Fontan outcome. Ann Thorac Surg 82:1260-1266.

Kogon BE, Plattner C, Leong T, et al. 2008. The bidirectional Glenn operation: a risk factor analysis for morbidity and mortality. J Thorac Cardiovasc Surg 136:1237-1242.

Lamberti JJ, Spicer RL, Waldman JD, et al. 1990. The bidirectional cavopulmonary shunt. J Thorac Cardiovasc Surg 100:22-30.

Lee MGY, Brizard CP, Galati JC, et al. 2014. Outcomes of patients born with single-ventricle physiology and aortic arch obstruction: the 26-year Melbourne experience. J Thorac Cardiovasc Surg 148:194-201.

Lui RC, Williams WG, Trusler GA, et al. 1993. Experience with the Damus-Kaye-Stansel procedure for children with Taussig-Bing hearts or univentricular hearts with subaortic stenosis. Circulation 88:II170-6.

Matitiau A, Geva T, Colan SD, et al. 1992. Bulboventricular foramen size in infants with double-inlet left ventricle or tricuspid atresia with transposed great arteries: influence on initial palliative operation and rate of growth. J Am Coll Cardiol 19:142-148.

Petrucci O, Khoury PR, Manning PB, Eghtesady P. 2010. Outcomes of the bidirectional Glenn procedure in patients less than 3 months of age. J Thorac Cardiovasc Surg 139:562-568.

Pridjian AK, Mendelsohn AM, Lupinetti FM, et al. 1993. Usefulness of the bidirectional Glenn procedure as staged reconstruction for the functional single ventricle. Am J Cardiol 71:959-962.

Rodefeld MD, Ruzmetov M, Schamberger MS, et al. 2005. Staged surgical repair of functional single ventricle in infants with unobstructed pulmonary blood flow. Eur J Cardiothorac Surg 27:949-955.

Tamisier D, Vouhe PR, Vernant F, et al. 1990. Modified Blalock-Taussig shunts: results in infants less than 3 months of age. Ann Thorac Surg 49:797-801.

Warrier G, Ch M, Dharan BS, et al. 2004. Bidirectional glenn operation in infancy. 159-163. 
The Heart Surgery Forum 2020-3299

Wernovsky G, Wypij D, Jonas RA, et al. 1995. Postoperative course and hemodynamic profile after the arterial switch operation in neonates and infants. A comparison of low-flow cardiopulmonary bypass and circulatory arrest. Circulation 92:2226-2235. 